\title{
The Effects of Two Weeks of Arm Crank Sprint Interval Training in Men with Chronic Spinal Cord Injury
}

\author{
Christopher R Harnish ${ }^{1}$, Roy T Sabo ${ }^{2}$, Jonathan A Daniels ${ }^{3}$ and Deborah Caruso ${ }^{4}$ \\ ${ }^{1}$ Department of Exercise Science, Shenandoah University, USA \\ ${ }^{2}$ Deptartment of Biostatistics, Virginia Commonwealth University, USA \\ ${ }^{3}$ Arizona University School of Medicine, USA \\ ${ }^{4}$ Department of Physical Medicine and Rehabilitation, USA
}

*Corresponding author: Christopher Harnish, PhD, Assistant Professor, Department of Exercise Science, Shenandoah University, Winchester, VA 22601, Tel: (540)692-6762, charnish@su.edu

\begin{abstract}
The purpose of this study was to examine the performance and metabolic effects of two weeks of Arm Crank Ergometry (ACE) Sprint Interval Training (SIT) in men with Spinal Cord Injury (SCl). Eight paraplegic males $50.5 \pm 9.0$ yo, $180.8 \pm 6.7 \mathrm{~cm}$ tall, $85.1 \pm 19.5 \mathrm{~kg}$, and $35.1 \pm 5.7 \%$ body fat completed three Oral Glucose Tolerance Tests (OGTTs) at baseline, 2 weeks later, prior to SIT, and $48 \mathrm{hrs} \mathrm{Post} \mathrm{SIT.}$ Six SIT sessions were performed on a Monark 891E ACE. Subjects cranked against $3.5 \%$ body mass for $30 \mathrm{sec}$, completing 4 sprints in session 1 , then $5,5,6,6$, and 7 sprints in the final session. All data are presented as means \pm SD with absolute change responses from baseline $\pm 95 \%$ confidence intervals and changes that failed to cross 0 considered significant. Peak and average power output increased across all subjects; peak power increased $11.9 \%$, while average power increased $9.9 \%$. AUC for neither glucose nor insulin significantly changed and ISI-Cederholm insulin sensitivity also failed to improve; OGTT change was $3.93(-23.4,31.3$ $95 \% \mathrm{Cl}$ ). However, post-SIT plasma Non-Esterified Fatty Acids (NEFA) AUC dropped 0.34 (-0.53, $-0.1695 \% \mathrm{Cl}$ ) mEq.L-1. In conclusion, two weeks of ACE SIT was effective at reducing NEFA in men with $\mathrm{SCl}$, but did not improve insulin sensitivity or glucose levels. These data indicate that ACE SIT may be an effective adjunct training modality for those with $\mathrm{SCl}$ and other non-ambulatory populations.
\end{abstract}

\section{Keywords}

Spinal cord injury, Sprint interval training, Insulin sensitivity, Arm crank ergometry, OGTT

\section{Abbreviations}

ACE: Arm Crank Ergometry; BLC: Blood Lactate Concentration; HIT: High-Intensity Interval Training; NEFA: Non-Esterified
Fatty Acid; OGTT: Oral Glucose Tolerance Test; SCI: Spinal Cord Injury; SIT: Sprint Interval Training; WIN: Wingate Training; WR: Work-to-Rest ratio

More than 6 million ( 2\%) Americans are living with a spinal cord injury or disorder [1]. Numerous studies have shown that up to half of persons with Spinal Cord Injury $(\mathrm{SCl})$ are obese, with nearly $70 \%$ of persons with $\mathrm{SCl}$ exhibiting two or more components of Metabolic Syndrome [2-5], with poor glucose and insulin regulation being most common [6]. Due to the close relationship between metabolic syndrome and both obesity and blood sugar management, mitigating either or both of these conditions is a priority for many populations, including $\mathrm{SCl}$.

Exercise is effective in reducing body fat [7] and improving many of the components of metabolic syndrome, like insulin resistance [8-10]. Exercise intensity, however, has been postulated as the critical component between improved mitochondrial function and Glucose Transporter (GLUT) 4 expression. Earnest [9] hypothesized that High-Intensity Interval (HIT), using efforts at > $80 \% \mathrm{VO}_{2 \text { Peak }}$, may offer a more powerful stimulus improving insulin sensitivity than moderate aerobic exercise, relating specifically to the aerobic metabolic processes (e.g., aerobic glycolysis, beta oxidation, and mitochondrial biogenesis). Similarly, Sprint Interval Training (SIT), which uses repeated "supramaximal" sprints above $\mathrm{VO}_{2}$

Citation: Harnish CR, Sabo RT, Daniels JA, Caruso D (2017) The Effects of Two Weeks of Arm Crank Sprint Interval Training in Men with Chronic Spinal Cord Injury. Int J Sports Exerc Med 3:059. doi. org/10.23937/2469-5718/1510059

Received: October 27, 2016; Accepted: June 08, 2017; Published: June 10, 2017

Copyright: (C) 2017 Harnish CR, et al. This is an open-access article distributed under the terms of the Creative Commons Attribution License, which permits unrestricted use, distribution, and reproduction in any medium, provided the original author and source are credited. 
Peak intensity [11], has also been studied extensively. For example, results by Gibala and others [12-14] have shown the powerful endurance-like impact that SIT has on oxidative capacity and metabolic function, while [13] showed that six weeks of SIT produced similar improvements in lipid levels, carbohydrate oxidation, and mitochondrial biogenesis compared to endurance training encompassing ten-times the total work expenditure (225 kJ vs. $2250 \mathrm{~kJ})$. More recently, [12] showed that six sessions of SIT improved insulin sensitivity by $37 \%$, as well as a significant reduction in Non-Esterified Fatty Acids (NEFA). Unfortunately, many populations cannot physically utilize leg ergometry [15], and may be limited to upper extremity exercise.

Upper extremity training is effective at improving central and peripheral fitness markers, and may transfer to lower extremity fitness $[7,16]$. Although training the upper extremities does improve force production and endurance of the muscles trained, intensity appears to be a critical factor in upper extremity fitness and transfer to lower extremity fitness [7]. Little is known, however, on the impact of specific Arm Crank Ergo Meter (ACE) interventions, including the adaptation of many HIT protocols, as well as SIT.

The purpose of this exploratory study was to examine the efficacy of six sessions of ACE SIT on sprint performance and metabolic factors, including insulin sensitivity and plasma NEFAs. We hypothesized that ACE SIT would improve sprint power output, as well improve peripheral insulin sensitivity (ISI-Cederholm), and reduce plasma NEFAs from pre-regimen levels in men with $\mathrm{SCl}$.

\section{Methods}

\section{Experimental approach to the problem}

In order to closely compare the effect of short-term SIT on persons with $\mathrm{SCl}$, the experimental protocol (Figure 1) was similar to previous SIT studies [12-14], utilizing the same work to rest ratio, which amounted to sessions lasting about $20 \mathrm{~min}$, with 3 sessions conducted over 2-weeks. Due to difficulty in recruiting and transporting persons with $\mathrm{SCl}$, we utilized a 2-week control period allowing each subject to act as their own control. During this period, two Oral Glucose Tolerance Tests (OGTT 1 and 2) were compared, while lifestyle and activity remained unchanged; a dietary recall was completed for the 24 -hr period prior to each OGTT. DXA body composition analysis was completed during the first test session. To minimize learning effects during training, subjects were familiarized with ACE Wingate training no less than 6 days prior to OGTT 2 to prevent any possible effect on blood test. Once completed, each participant began their sprint training within 36 -hours after the second pre-training OGTT2.

A total of six sprint training sessions took place over a period of 2 weeks. A final post post-training OGTT3 was performed between 48 and $60 \mathrm{hrs}$ of the final training session; this time period matched previously published work [12]. The Area Under the Curve (AUC) was calculated for glucose, insulin, and NEFA's for pre and post blood testing. Utilizing this protocol, we surmised that baseline and pre-training blood variables would not change, while those same variables would change as shown in other studies [12-14] after six sessions of SIT. Enrollment and testing began in June 2010 and were concluded approximately 18 mo later.

\section{Subjects}

All experimental protocols were reviewed and approved by the Hunter Holmes McGuire VAMC institutional review board and comply with the Declaration of Helsinki. All participants were actively recruited through word of mouth, flyers and call lists, with individuals being both veterans visiting the Hunter Holmes McGuire Veterans Administration Medical Center (VAMC) and individuals living in the greater Richmond, Virginia (USA) Community. Subjects were enrolled in the study following completion of the McGuire IRB approved informed consent document. A detailed medical history and American College of Sports Medicine (ACSM) risk factor assessment was performed for all participants and each subject was reviewed by an $\mathrm{SCl}$ board-certified physician. Only individuals considered paraplegics (i.e., T1-L2) AIS (American Spinal Injury Association Classification)-A through $D$ for 3 months or longer were considered for the study. Individuals classified as High Risk based on established ACSM criteria, persons with known orthopedic limitations, diabetes mellitus (fasting glucose $>126$ or HgbA1c > 7.0), hypothyroidism, renal disease, uncontrolled autonomic dysreflexia, recent (within 3 months) deep vein thrombosis, or pressure ulcers $>$ Stage II were excluded from participation. A total of 12 sedentary men with T1-T10 SCl initiated the study,

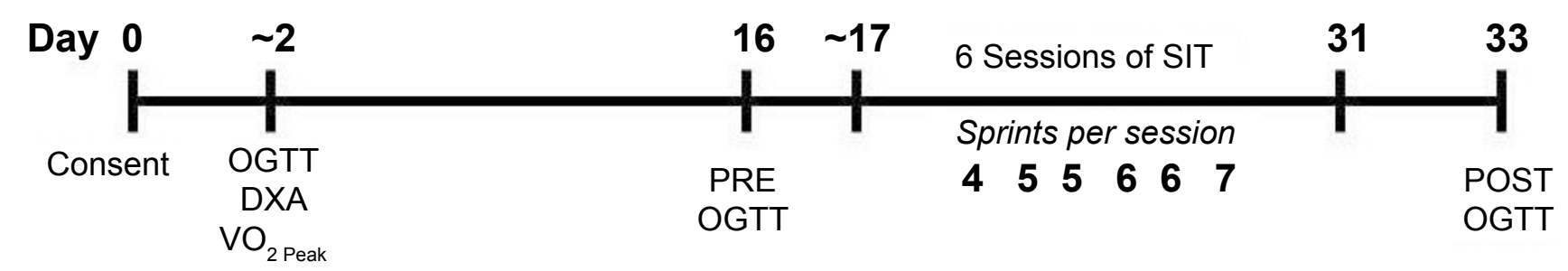

Figure 1: Experimental design for 2 weeks of sprint interval training in individuals with SCI. 
with eight completing the entire protocol; subject withdrawal was due to lack of transportation to the laboratory, with three withdrawing prior to beginning SIT, and one after their second sprint session.

\section{Procedures}

Subjects were instructed to maintain their usual diet throughout the study and to consume similar meals the day before each OGTT; a standard 24-hr dietary recall form for the day prior to the OGTT, with subsequent forms completed preceding each OGTT. Subjects were also instructed to consume 8-16 oz of water prior to their arrival for testing. During the pre-training control period and after the first OGTT, each subject completed one $\mathrm{VO}_{2 \text { Peak }}$ testing and this was used to assess physical readiness and determine maximum 2 min power. Testing was performed using a Lode upper extremity ergo meter (Electro-Med Corporation, Flint, MI). Subjects performed the test in their own stabilized wheelchair with appropriate seating, trunk support, leg wraps, abdominal binder and protective hand mitts provided. Each subject cranked at 70 RPM against no resistance for 3 min before work rate was increased by $15 \mathrm{~W}$ every 3 min until volitional exhaustion was reached. $\mathrm{VO}_{2}$ and $\mathrm{HR}$ were measured continuously using a Cosmed $\mathrm{K}^{4} \mathrm{~b}^{2}$ (Cosmed USA, Inc. Chicago, IL) and Polar RS 400 HR monitor (Polar Electro Inc., Lake Success, NY), respectively, and stored for later analysis. Subjects were familiarized with the training protocol no less than 5 days prior to training to mitigate any influence on the Pre OGTT. Each subject repeated this test after their final OGTT.

Sprint training was performed using a mechanically braked Monark 891E arm crank ergo meter (Fitness Superstore, Inc., Concord, CA), while sitting in their own stabilized wheelchair with appropriate seating, trunk support, leg wraps, abdominal binder and protective hand mitts provided. Following $10 \mathrm{~min}$ of unloaded warm-up at 30-50 rpm, each subject cranked as fast as possible for $30 \mathrm{sec}$ against a resistance equivalent to $0.035 \mathrm{~kg} / \mathrm{kg}(3.5 \%)$ body weight a resistance shown to be suitable for thoracic level persons with $\mathrm{SCl}$ in the pilot phase of the study [16]. Subjects cranked while being provided with vigorous verbal encouragement throughout each sprint. Peak and mean power (W) and total work (kJ) were recorded using the software provided with the ergo meter. Each sprint was followed by $\sim 5$ min of rest or slow reverses pedaling. The initial training session included four sprints progressing to five sprints in sessions 2 and 3, six sprints in sessions 4 and 5, and finally seven sprints in the final session. Sessions were separated by at least $48 \mathrm{hrs}$, but no more than $72 \mathrm{hrs}$ (i.e., one weekend). During sessions 1 and 6, $5 \mu$ blood lactate samples were taken from the subjects earlobe using a small plastic lancet and immediately analyzed using a Lactate Scout Analyzer (Sports Resource Group, Hawthorne, NY). Samples were taken prior to training, after sprint 1, 4 and 7 (during session 6), and 1 and 3 min post exercise. Each training session lasted approximately $30 \mathrm{~min}$. Due to the increase in the number of sprints performed during training; the relative improvement in sprint work capacity was compared using the total kJ expenditure.

All OGTT's were performed between 0800 and 1000 hours, and each subject's tests were conducted at the same time $( \pm 5 \mathrm{~min})$ of the first test. Subjects refrained from performing any strenuous physical activity for a period of $72 \mathrm{hrs}$ prior to baseline and Pre OGTT, and arrived at the laboratory following at least a $12 \mathrm{hr}$ overnight fast. Venous blood samples ( $10 \mathrm{ml})$ were collected by venipuncture before ingestion, and at 60, 90 and 120 min after ingestion of $75 \mathrm{~g}$ glucose (NOW Foods, Bloomingdale, IL) dissolved in $100 \mathrm{ml}$ of water. Plasma was separated by centrifugation (15 min at $7000 \mathrm{rpm}$ ) and stored at $-20^{\circ} \mathrm{C}$ until analysis of glucose, insulin, and NEFA concentrations. All samples were measure in duplicate. Plasma glucose concentrations were measured using an auto-analyzer glucose oxidase method, while plasma insulin concentrations were determined by ELISA (R \& D Systems, Inc, Minneapolis, MN). Plasma NEFA concentrations were determined by a colorimetric assay (Wako Chemicals, Germany) using a modified protocol. Briefly $3.75 \mu$ of plasma samples and standards of known concentration were pipetted into a 96-well plate $75 \mu \mathrm{l}$ of color reagent $A$ were added to each well and incubated at $37{ }^{\circ} \mathrm{C}$ for $10 \mathrm{~min} .150 \mu \mathrm{l}$ of colour reagent $B$ were added and incubated for a further $10 \mathrm{~min}$ at 37 ${ }^{\circ} \mathrm{C}$. The plate was then removed from the incubator and allowed to cool to room temperature prior to the absorbance being read at $550 \mathrm{~nm}$. In order to assess normal intra-individual variation in response to an OGTT over a period of several weeks as used in the present study, all subjects performed the initial OGTT prior to any testing in week 0 and compared to the Pre training test at week 2. Coefficients of Variation (CV) for glucose, insulin, and NEFA were $5.6 \%, 6.1 \%$, and $6.6 \%$, respectively.

\section{Statistical analyses}

Data were analyzed using Jump 13.0 software (SAS Institute Inc., Cary, NC). All data are presented as means \pm SD. The Area Under the Curve (AUC) was calculated using the trapezoidal rule and peripheral Insulin Sensitivity $\left(\mathrm{S}_{\mathrm{i}}\right)$ was estimated using ISI-Cederholm.

Cederholm Si $=\frac{75000+(G 0-G 120) \times 1.15 \times 180 \times 0.19 \times \frac{B W}{120} \times \text { Gmean } \times \log (\text { Imean })}{1000}$

$\mathrm{BW}=$ body weight, $\mathrm{G}_{0}$ and $\mathrm{G}_{120}$ are plasma glucose concentration at 0 and $120 \mathrm{~min}\left(\mathrm{mmol}^{\mathrm{I}} \mathrm{I}^{-1}\right.$, and $\mathrm{I}_{\text {mean }}$ and $\mathrm{G}_{\text {mean }}$ are the mean insulin $\left(\mathrm{Mu} . \mathrm{I}^{-1}\right)$ and glucose $\left(\mathrm{mmol} . \mathrm{I}^{-1}\right.$ ) concentrations during the OGTT.

Dependent variables, like insulin sensitivity, glucose, insulin, and NEFA AUC, as well as sprint performance markers are reported as absolute values and changes from baseline. Data were analyzed using absolute change responses from baseline $\pm 95 \%$ confidence inter- 
Table 1: Summary of physiological and performance changes before and after six Sprint Interval Training (SIT) sessions. Note, $\mathrm{VO}_{2 \text { Peak }}$ testing was conducted prior to and after SIT. SIT power and work data were taken from the first four sprints of each session. ${ }^{*} 95 \% \mathrm{Cl}$ changes that failed to cross 0 (i.e., 0 change) were considered significant with a significant increase above initial values.

\begin{tabular}{|l|l|l|l|l|}
\hline Measure & Mean (SD) & Mean (SD) & Change Mean (95\% Cl) & \% Change Mean (95\% CI) \\
\hline & Pre-training & Post-training & & \\
\hline $\mathrm{VO}_{2 \text { Peak }}\left(\mathrm{L} \cdot \mathrm{min}^{-1}\right)$ & $1.41(0.44)$ & $1.50(0.49)$ & $0.12(0.04,0.17)^{*}$ & $7.6(1.5,11.1)^{*}$ \\
\hline $\mathrm{VO}_{2 \text { Peak }}$ Power (W) & $84.4(25.3)$ & $97.5(26.6)$ & $13.1(5.1,21.2)^{*}$ & $17.9(3.7,30.4)^{*}$ \\
\hline & SIT Session 1 & SIT Session 6 & & \\
\hline Peak Power (W) & $274.7(72.2)$ & $307.0(80.8)$ & $34.1(13.3,54.8)^{*}$ & $11.9(4.3,19.5)^{*}$ \\
\hline Mean Power (W) & $188.7(47.8)$ & $207.2(54.4)$ & $23.7(7.3,40.1)^{*}$ & $9.9(2.2,17.7)^{*}$ \\
\hline$\% \mathrm{VO}_{2 \text { Peak }}$ Power & $231.7(57.3)$ & $217.8(49.5)$ & $-13.8(-29.1,1.5)$ & -- \\
\hline Work (kJ) & $5.66(1.44)$ & $6.18(1.63)$ & $0.69(0.22,1.15)^{*}$ & $12.0(4.6,19.4)^{*}$ \\
\hline BLC (mM) & $12.0(2.5)$ & $11.3(2.3)$ & $0.8(-1.9,0.2)$ & -- \\
\hline
\end{tabular}

Table 2: Summary of Pre- and Post-Test Areas under Curves for glucose, insulin, and NEFA, and ISI-Cederholm changes before and after six Sprint Interval Training (SIT) sessions. " $95 \% \mathrm{Cl}$ changes that failed to cross 0 (i.e., 0 change) were considered significant with a significant increase above initial values.

\begin{tabular}{|l|l|l|l|}
\hline Measure & Average OGTT 1 \& 2 Mean (SD) & OGTT 3 Mean (CD) & Change Mean (95\% CI) \\
\hline Glucose $\left({\left.\mathrm{mg} \cdot \mathrm{dL}^{-1}\right)}^{-1}\right.$ & $76.6(10.4)$ & $76.2(18.1)$ & $-0.4(-11.8,11.0)$ \\
\hline Insulin $\left(\mathrm{mU} \cdot \mathrm{I}^{-1}\right)$ & $168.5(78.5)$ & $139.0(52.9)$ & $-29.5(-91.3,32.4)$ \\
\hline NEFA $\left(\mathrm{mEq} \mathrm{L}^{-1}\right)$ & $1.35(0.4)$ & $1.01(0.46)$ & $-0.34(-0.53,-0.16)^{*}$ \\
\hline ISI-Cederholm & $169.4(29)$ & $175.2(46.3)$ & $3.93(-23.4,31.3)$ \\
\hline
\end{tabular}

vals; $95 \% \mathrm{Cl}$ changes that failed to cross 0 (i.e., 0 change) were considered significant.

\section{Results}

All data were reviewed and normally distributed. Subjects were $50.5 \pm 9.0$ yo, $180.8 \pm 6.7 \mathrm{~cm}$ tall, $85.1 \pm 19.5$ $\mathrm{kg}$ and $35.1 \pm 5.7 \%$ body fat. With the exception of one subject, all were diagnosed as AIS-A spinal cord injury. Participants tolerated the SIT well and completed all sessions over the 2-week trial; SIT intensity was conducted at over $200 \%$ of $\mathrm{VO}_{2 \text { peak }}$ power output. Sprint data from sessions 1 and 6 , as well as pre and post training $\mathrm{VO}_{2 \text { peak }}$ testing are summarized in Table 1 . Across all subjects, both peak and average power output increased across all subjects; peak power increased $11.9 \%$, while average power increased $9.9 \%$. The average work across the first four sprints of each session also increased $12 \% . \mathrm{VO}_{2 \text { Peak }}$ and the associated power output increased $7.6 \%$ and $17.9 \%$, respectively. SIT training proved less impactful on the metabolic markers examined in this study, with only NEFA's showing a significant smaller AUC posttest; $-0.34(-0.53,-0.1695 \% \mathrm{Cl}) \mathrm{mEq} \mathrm{L}^{-1}$. Table 2 summarizes the changes in metabolic markers for this study. While ISI-Cederholm values did not change after 2-weeks of SIT, it must be noted that the CV between OGTT 1 and 2 was $22.9 \%$, indicating a wide day-to-day variability in testing. Further, the variability could not be explained by differences in dietary intake. Dietary analysis indicated subjects consumed $2009.8+785.6 \mathrm{kcal}$, consisting of $251.6 \pm 114 \mathrm{~g} \mathrm{CHO}, 75.8 \mathrm{~g} \pm 41.7 \mathrm{~g}$ fat, and $78.0 \pm 29.6$ $\mathrm{g}$ protein, without significant variations between tests.

\section{Discussion}

The purpose of this study was to evaluate the efficacy of arm crank SIT for paraplegic men. Based on the research available for leg sprinting [12-14] and upper extremity training $[7,17]$ at the time of the study, we speculated that ACE SIT could improve sprint performance and metabolic factors in men with chronic $\mathrm{SCl}$. Following 2-week of arm crank SIT, there were significant improvements in performance measures including both sprint and aerobic measures. While, SIT failed to produce significant improvement in glucose tolerance or insulin sensitivity, we did see a significant reduction in NEFA curves. These findings suggest that SIT can have substantial impact on performance indices but not carbohydrate metabolism in men with $\mathrm{SCl}$.

The untrained male participants in this study showed a high training capacity with an improvement in sprint power output and average work of about $10 \%$, and an improvement in aerobic power output of nearly $20 \%$. Contemporary performance research typically considers a $1 \%$ improvement as the least meaningful improvement for events lasting 30 sec or less [18], indicating that our findings represent almost certain improvements in performance that were consistent across all subjects. While the increased in aerobic power are most likely tied to improved motor unit recruitment [11], the greater overall work capacity would improve overall upper extremity power, an important fitness component for wheelchair propulsion and activities of daily living [1922]. For example, loss of upper extremity strength and power significantly increases one's risk for premature mortality [21], and strength and power have a profound influence on one's ability to perform activities of daily living and wheelchair propulsion [19,22]. SIT can also provide enhanced power for wheelchair propulsion, reducing physical strain and improving one's ability to perform activities of daily living; all essential aspects to long-term health. 
It is well documented that persons with $\mathrm{SCl}$ suffer a multitude of metabolic disruptions [2-6,23], therefore improvements in metabolic markers are an important outcome for exercise. Unfortunately, SIT failed to influence carbohydrate metabolism or insulin sensitivity. Our data indicate that neither resting glucose, insulin, nor their AUC's were reduced following SIT; likewise, peripheral insulin sensitivity also failed to show improvement. While earlier research indicated that SIT could improve insulin sensitivity, acute SIT using either short or long rest periods [24] failed to improve insulin sensitivity among healthy untrained individuals. In fact, 2017 meta-analysis [25] suggests that neither short nor long-term SIT improves blood glucose-related control mechanisms. Add to this the high day-to-day variability $(23 \% \mathrm{CV})$ in glucose and insulin response was too great to overcome with upper extremity exercise alone. Our subjects reflected typical body fat levels (>30\%) seen after $\mathrm{SCl}$, and $\mathrm{ACE}$ has been shown to produce lower work outputs [4]. Moreover, other researchers have encountered similar problems using the OGTT (Personal Communication, T. Ryan 2012), and even the Intravenous Glucose Tolerance Test (IVGTT) data (unpublished) from our lab suggest that it may not be an ideal test to assess metabolic improvements for persons with $\mathrm{SCl}$. In contrast, [12] reported a CV in glucose AUC of less than $5 \%$. These variations could not be accounted for by diet, which was monitored and showed minimal variations 24-hr prior to each OGTT.

In contrast to glucose and insulin, we did see significant reduction in plasma NEFA AUC indicating that some blood lipids may be influenced by SIT, and that NEFA's may be a useful metabolic training marker in persons with $\mathrm{SCl}$ [17]. Further, our 2-hour trends in NEFA levels relate closely to those published by Babraj, et al. [12] following lower extremity sprints (Figure 2). Elevated plasma NEFA levels have been shown to directly and indirectly impair insulin sensitivity $[26,27]$; persons with $\mathrm{SCl}$ show elevated NEFA levels $[3,6]$, making reductions an important outcome with training. A reduction in NEFA in the blood could indirectly improve insulin sensitivity [27]. Moreover, long-term training utilizing SIT and resistance training could significantly impact factors other than just glucose metabolism. For example, [17] demonstrated that three months of combined aerobic and resistance circuit training significantly improves both cardiovascular fitness and blood lipid profiles in men with $\mathrm{SCl}$, while Jacobs [20] demonstrated impressive cardiovascular and power enhancements from heavy resistance training alone.

Application of interval training for persons with $\mathrm{SCl}$ remains lacking. Our data suggest that high-intensity interval and sprint interval training can be applied using established training principles and may afford an additional modality for training and overall improvement in quality of life, regardless of metabolic improvement. For example, we recently published a case report [28] of one of the individuals completing this SIT study, a T1 AIS-A male, took part in a 12-wk mixed interval ACE training program consisting of only three 30 min training sessions each week. Over the course of the 3-month training period the individual saw a $50 \%$ increase in $\mathrm{VO}_{2}$ Peak and a 56\% improvement in 30 min arm crank time trial performance. Clearly, more work is needed in this

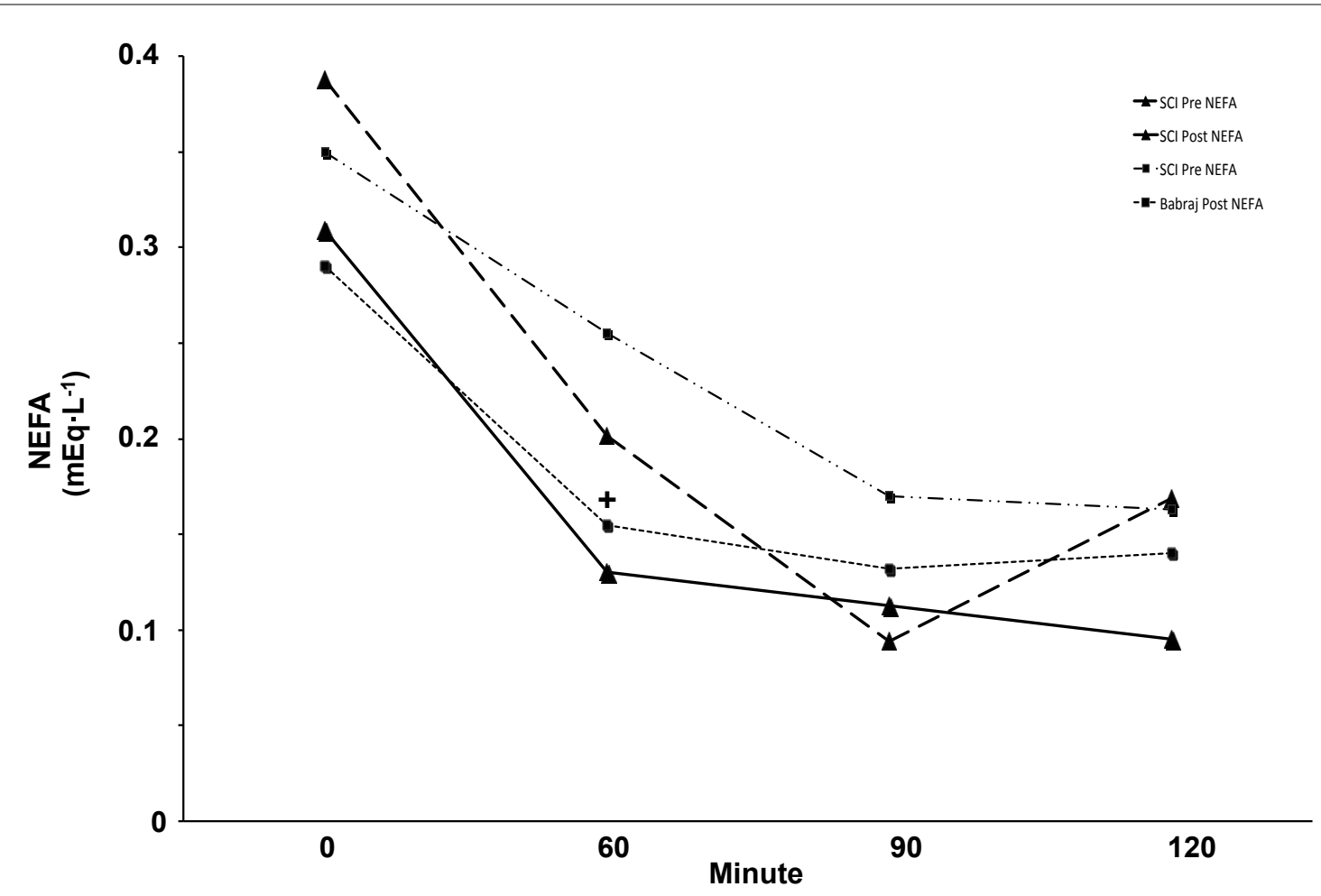

Figure 2: Illustrative comparison of NEFA values Pre and Post training for our SCl group (Triangle) and means reported by Babraj, et al. (Square)*. Both study groups showed a significant in AUC. *Adapted with permission. 
area to optimize training programs for persons with $\mathrm{SCl}$ care should be taken when implementing SIT with each individual.

This study demonstrates that just 2-wk of SIT can have a substantial impact on upper extremity power in men with $\mathrm{SCl}$. Nonetheless, we recognize that the major limitation in this study is the relatively small sample size of diverse individuals with $\mathrm{SCl}$. A larger study, perhaps with greater dietary controls, including prepared meals, may yield greater metabolic improvements. And while the latest review of research suggests that SIT may offer limited value for managing blood sugar, physical performance improvements are in many ways equally important, as they improve individual independence and work capacity, making further participation in activity more likely. More research is needed to elucidate the effects of longer duration interval programs for those with $\mathrm{SCl}$.

\section{Acknowledgements}

All research was made possible with the funding support of VHA RR\&D Merit Grants B3918R and B6757R247, and Virginia Commonwealth University's Center for Clinical and Translational research grant \#UL1TR000058, NCATS (National Center for Advancing Translational Sciences), NIH. We wish to thank Allison Keeley for her help in collecting and analyzing the data, and greatly appreciate the effort of all our subjects during the strenuous training protocol.

\section{Conflict of Interest Statement}

We report no known conflicts of interest.

\section{Ethical Statement}

All experimental protocols were reviewed and approved by the Hunter Holmes McGuire VAMC institutional review board and comply with the Declaration of Helsinki.

\section{Funding Sources}

All research was made possible with the funding support of VHA RR\&D Merit Grants B3918R and B6757R247, and Virginia Commonwealth University's Center for Clinical and Translational research grant \#UL1TR000058, NCATS (National Center for Advancing Translational Sciences), NIH.

\section{References}

1. Christopher, Dana Reeve Foundation (2009) One Degree of Separation: Paralysis and Spinal Cord Injury in the United States.

2. DeVivo MJ, Schechuk RM, Stover SL, Black KL, Go BK (1992) A cross-sectional study of the relationship between age and current health status for person with spinal cord injuries. Paraplegia 30: 820-827.

3. Gater DR Jr (2007) Obesity after spinal cord injury. Phys Med Rehabil Clin N Am 18: 333-351.

4. Gater DR (2009) Spinal Cord Injury. In: Ehrman JK, Gordon PM, Visich PS, Keteyian SJ, Clinical Exercise Physiology. $\left(2^{\text {nd }}\right.$ edn $)$, Human Kinetics, Champaign.
5. Lavela SL, Weaver FM, Goldstein B, Chen K, Miskevics S, et al. (2006) Diabetes mellitus in individuals with spinal cord injury or disorder. J Spinal Cord Med 29: 387-395.

6. Wilt TJ, Carlson KF, Goldish GD, MacDonald R, Niewoehner C, et al. (2008) Carbohydrate and lipid disorders and relevant considerations in persons with spinal cord injury. Evid Rep Technol Assess (Full Rep) 163: 1-95.

7. Tordi N, Belli A, Mougin F, Rouillon JD, Gimenez M (2001) Specific and transfer effects induced by arm or leg training. Int J Sports Med 22: 517-524.

8. Colberg SR, Grieco CR (2009) Exercise in the treatment and prevention of diabetes. Curr Sports Med Rep 8: 169175.

9. Earnest CP (2008) Exercise interval training: an improved stimulus for improving the physiology of pre-diabetes. Med Hypotheses 71: 752-761.

10. Hawley JA, Gibala MJ (2009) Exercise intensity and insulin sensitivity: how low can you go? Diabetologia 52: 17091713.

11. Maclnnis MJ, Gibala MJ (2016) Physiological adaptations to interval training and the role of exercise intensity. J Physiol 595: 2915-2930.

12. Babraj JA, Vollaard NB, Keast C, Guppy FM, Cottrell G, et al. (2009) Extremely short duration high intensity interval training substantially improves insulin action in young healthy males. BMC Endocr Disord 9: 3.

13. Burgomaster KA, Howarth KR, Phillips SM, Rakobowchuk M, Macdonald MJ, et al. (2008) Similar metabolic adaptations during exercise after low volume sprint interval and traditional endurance training in humans. J Physiol 586: 151-160.

14. Gibala M (2011) Use of high intensity training in metabolic syndrome patients. ACSM Annual Meeting, Denver, 43-45.

15. Hardy SE, Kang Y, Studenski SA, Degenholtz HB (2011) Ability to walk $1 / 4$ mile predicts subsequent disability, mortality, and health care costs. J Gen Intern Med 26: 130-135.

16. Jacobs PL, Mahoney ET, Johnson B (2003) Reliability of arm Wingate Anaerobic Testing in persons with complete paraplegia. J Spinal Cord Med 26: 141-144.

17. Nash MS, Jacobs PL, Mendez AJ, Goldberg RB (2001) Circuit resistance training improves the atherogenic lipid profiles of persons with chronic paraplegia. J Spinal Cord Med 24: $2-9$.

18. Paton CD, Hopkins WG (2005) Combining explosive and high-resistance training improves performance in competitive cyclists. J Strength Cond Res 19: 826-830.

19. Janssen TW, van Oers CA, Rozendaal EP, Willemsen EM, Hollander AP, et al. (1996) Changes in physical strain and physical capacity in men with spinal cord injuries. Med Sci Sports Exerc 28: 551-559.

20. Jacobs PL (2009) Effects of resistance and endurance training in persons with paraplegia. Med Sci Sports Exerc 41: 992-997.

21. Metter EJ, Talbot LA, Schrager M, Conwit RA (2004) Arm-cranking muscle power and arm isometric muscle strength are independent predictors of all-cause mortality in men. J Appl Physiol (1985) 96: 814-821.

22. Zoeller RF Jr, Riechman SE, Dabayebeh IM, Goss FL, Robertson RJ, et al. (2005) Relation between muscular strength and cardiorespiratory fitness in people with thoracic-level paraplegia. Arch Phys Med Rehabil 86: 1441-1446. 
23. Rajan S, McNeely MJ, Warms C, Goldstein B (2008) Clinical assessment and management of obesity in individuals with spinal cord injury: a review. J Spinal Cord Med 31: 361-372.

24. Harnish CR, Sabo RT (2016) Comparison of Two Different Sprint Interval Training Work-to-Rest Ratios on Acute Inflammatory Responses. Sports Med Open 2: 20.

25. Batacan RB, Duncan MJ, Dalbo VJ, Tucker PS, Fenning AS (2016) Effects of high-intensity interval training on cardiometabolic health: a systematic review and meta-analysis of intervention studies. Br J Sports Med 51: 494-503.

26. Grundy SM (2004) Obesity, metabolic syndrome, and cardiovascular disease. J Clin Endocrinol Metab 89: 2595-2600.

27. Kahn SE, Hull RL, Utzschneider KM (2006) Mechanisms linking obesity to insulin resistance and type 2 diabetes. Nature 444: 840-846.

28. Harnish CR, Daniels JA, Caruso D (2017) Training response to high-intensity intervaltraining in a 42-year-old man with chronicspinal cord injury. J Spinal Cord Med 40: 246-249. 